\section{Avances en el tratamiento de cáncer de próstata resistente a la castración: énfasis en nuevas terapias hormonales}

\author{
ALEJANDRO BERLIN ${ }^{1,2,3}$, MARIO I. FERNÁNDEZ ${ }^{4,5}$
}

\section{Advances in the treatment of castration-resistant prostate cancer: emphasis in new hormonal therapies}

\begin{abstract}
Prostate cancer represents the second cancer-related cause of death in North American and Chilean men. The main treatment for incurable stages of disease is surgical or pharmacological castration. However, with time and despite the addition of anti-androgens, the disease progresses to a clinical state that has been commonly referred to as "hormone refractory". In recent years, the concept of hormone refractoriness has been challenged and replaced by "castration resistance", acknowledging that further and optimal hormonal manipulation can be attained, beyond achieving testosterone levels at castration range. The purpose of this review is to summarize the recent therapeutic breakthroughs in the management of metastatic castrate resistant prostate cancer ( $m C R P C)$, with greater emphasis in the newer hormonal therapy agents such as Abiraterone and Enzalutamide. Future combination and sequential treatment strategies are contextualized in the current era of personalized cancer medicine and genomic characterization of prostate cancer.
\end{abstract}

(Rev Med Chile 2015; 143: 223-236)

Key words: Abiraterone; Enzalutamide; Prostatic neoplasms, castrationresistant.

\author{
'Servicio de Radioterapia, Clínica \\ Alemana de Santiago. Santiago, \\ Chile. \\ ${ }^{2}$ Radiation Medicine Program, \\ Princess Margaret Cancer Centre. \\ Toronto, Canadá. \\ 3Department of Radiation \\ Oncology, University of Toronto. \\ Toronto, Canadá. \\ ${ }^{4}$ Servicio de Urología, Clínica \\ Alemana de Santiago. Santiago, \\ Chile. \\ ${ }^{5}$ Centro de Genética y Genómica, \\ Facultad de Medicina Clínica \\ Alemana-Universidad del \\ Desarrollo. Santiago, Chile. \\ Recibido el 7 de julio de 2014, \\ aceptado el 10 de noviembre \\ de 2014. \\ Correspondencia a: \\ Dr. Alejandro Berlin \\ Av. Vitacura 5951 \\ Vitacura, Santiago, Región \\ Metropolitana, Chile. \\ aberlin@alemana.cl \\ Alejandro Berlin, MD, MSc \\ 610 University Ave \\ 5th Floor-DRO \\ Toronto, Canadá. M5T 2M9 \\ alejandro.berlin@rmp.uhn.ca
}

E 1 cáncer de próstata $(\mathrm{CP})$ constituye, en hombres, el segundo diagnóstico oncológico más frecuente y una de las principales causas de mortalidad general y cáncer-específica ${ }^{1}$. En Chile, el CP representa la segunda causa de muerte masculina por cáncer ${ }^{2}$. Desde los trabajos originales de Huggins y Hodges en $1941^{3}$, el tratamiento central en las etapas incurables ha sido la castración quirúrgica o farmacológica. Sin embargo, luego de un promedio de 2 a 8 años de terapia $^{4,5}$ (dependiendo de la etapa de la enfermedad y momento de inicio de la terapia hormonal) la enfermedad progresa, a pesar de la adición de medicamentos antiandrogénicos, alcanzándose el estadio que comúnmente se denominaba "hor- monorefractario". No obstante, los últimos años se han caracterizado por múltiples avances terapéuticos, sustentados en un mejor entendimiento de la fisiopatología de la enfermedad. El concepto de hormonorefractariedad ha sido reemplazado por "resistente a la castración", reconociendo el hecho que mayor y mejor manipulación hormonal puede ser lograda más allá de la obtención de niveles plasmáticos de testosterona en rango de castración $(<50 \mathrm{ng} / \mathrm{dL})$.

La primera terapia en ser aprobada para el manejo del CP resistente a la castración (CPmRC) fue la mitoxantrona, basado en estudios que demostraron beneficios paliativos pero no traducidos en mejorías de sobrevida ${ }^{6,7}$. Posteriormente, 
docetaxel demostró beneficios en sobrevida global (incremento medio de 2-3 meses) $^{8-10}$. De acuerdo a esto, hasta hace poco, la recomendación de manejo inicial en el CPmRC incluía como primera línea el uso de docetaxel y prednisona $\left(75 \mathrm{mg} / \mathrm{m}^{2}\right.$ cada 3 semanas y $10 \mathrm{mg}$ al día, respectivamente) para pacientes en condiciones de recibir terapia citotóxica ${ }^{11}$. Sin embargo, en forma invariable, los pacientes sufren progresión de la enfermedad durante o posterior a esta terapia mediante diferentes mecanismos de resistencia inherentes o adquiridos ${ }^{12,13}$. Más allá de eso, hasta el año 2010, ninguna de las opciones terapéuticas de segunda línea había demostrado impacto en la sobrevida global. Afortunadamente, la intensa búsqueda de nuevos y mejores agentes terapéuticos para el tratamiento del CPmRC ha tenido una productividad sin precedentes en los últimos años, que se ha reflejado en las recientes aprobaciones por la FDA (Food and Drug Administration) y la EMA (European Medicines Agency) de abiraterona acetato (AA), enzalutamida (previamente MDV3100), cabazitaxel, radio-223 y sipuleucel-T como nuevos agentes para el tratamiento del CPmRC. El objetivo del presente artículo es resumir los recientes avances en las opciones terapéuticas para el manejo del CPmRC, con énfasis en las nuevas terapias hormonales.

\section{Esteroidogénesis y Receptor Androgénico en CPmRC}

A pesar de los bajos niveles de testosterona plasmática, en un porcentaje significativo, el CP permanece sujeto a la acción de vías de señalización celular dependientes del receptor androgénico (RA). Durante el estado de castración, esta activación puede ocurrir mediante múltiples mecanismos ${ }^{14,15}$ : síntesis androgénica suprarrenal, síntesis intratumoral de novo, sobreexpresión del RA (amplificación del locus del gen AR y/o sobrerregulación transcripcional), generación de variantes activas mediante splicing alternativo, activación ligando-independiente del receptor (ej. mediante citoquinas o factores de crecimiento) y aumento en la captación de hormonas esteroidales circulantes. Por todo lo anterior, la esteroidogénesis y el RA continúan siendo un objetivo importante para el desarrollo de nuevas terapias para el CP (Figura 1; Tabla 1) ${ }^{16}$.

\section{Acetato de Abiraterona: esteroidogénesis como blanco terapéutico}

El acetato de abiraterona (AA) es una prodroga oral, que se transforma en el compuesto activo abiraterona. Su mecanismo de acción es la inhibición irreversible del complejo enzimático CYP-17, bloqueando dos reacciones cruciales de la síntesis androgénica: la conversión de pregnenolona en 17-OH pregnenolona y la subsecuente conversión de ésta en dehidroepiandrosterona (DHEA) ${ }^{17}$. La inhibición del complejo CYP-17 también bloquea la producción de corticoesteroides y un desequilibrio hacia la producción excesiva de mineralocorticoides (Figura 1). Para contrarrestar estos últimos dos fenómenos, se administran simultáneamente corticoides exógenos (prednisona $10 \mathrm{mg}$ al día). De esta manera, las alteraciones en la esteroidogénesis y el uso concomitante de prednisona, explican varios de los efectos secundarios del tratamiento (Tabla 2).

AA fue evaluada en un estudio controlado aleatorio doble-ciego fase III(COU-AA-301) que incluyó pacientes con CPmRC en progresión de enfermedad post-docetaxel, con niveles hormonales de castración (testosterona $<50 \mathrm{ng} / \mathrm{dL}$ ) y buen estado funcional (ECOG PS 0-2). Los pacientes fueron asignados (en razón 2:1) a AA $(1.000 \mathrm{mg}$ al día) y prednisona ( $5 \mathrm{mg}$ x 2 veces al día) versus placebo y prednisona (misma dosis). La variable principal del estudio fue sobrevida global, con variables secundarias de progresión, respuesta y calidad de vida. El tratamiento se mantuvo hasta la progresión clínica o radiológica. El incremento aislado de APE (antígeno prostático específico) no fue criterio suficiente para interrumpir la terapia. Luego del análisis interino, la rama activa (AA/ prednisona) presentó una sobrevida global de 14,8 versus 10,9 meses en el grupo placebo/prednisona (HR 0,65: IC 95\% 0,54-0,77; p < 0,001) $)^{18}$. Basados en esta observación, el Comité Independiente de Monitorización determinó que el estudio se abriera permitiendo a los pacientes en la rama de placebo pasar al grupo de tratamiento activo por los beneficios evidentes y precozmente observados. El análisis final, luego de una mediana de 20,2 meses de seguimiento y ocurridos 775 eventos, confirmó el impacto positivo en sobrevida global (Tabla 1). Este beneficio se reflejó también en todas las variables secundarias ${ }^{19}$. Un análisis separado, observó que el uso de AA/prednisona se 


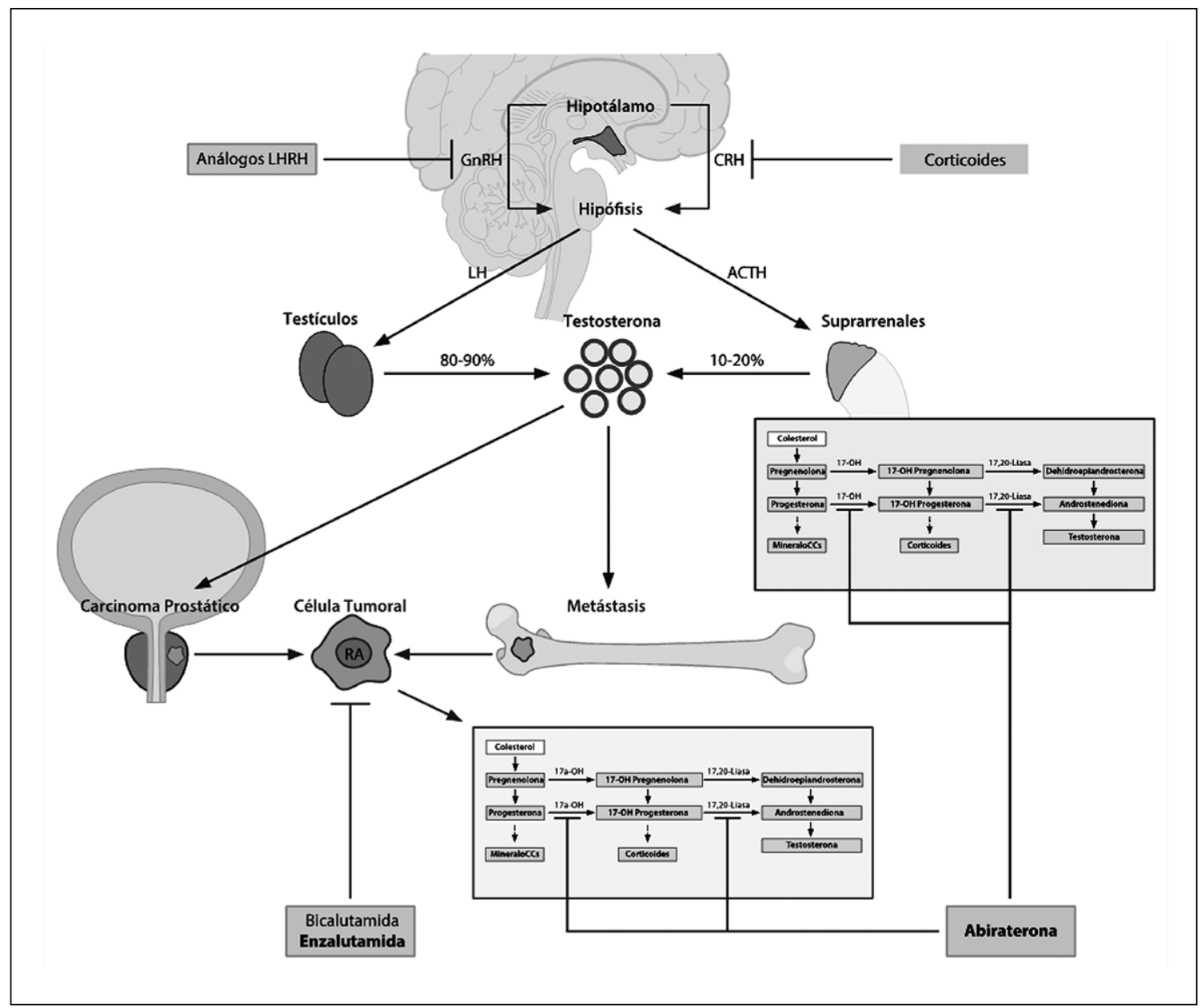

Figura 1. Representación esquemática de los sitios de acción de las terapias hormonales en cáncer de próstata resistente a la castración.

asoció además a mejoría sintomática relacionada a metástasis óseas y a retardo en la ocurrencia de dolor y eventos relacionados a estas metástasis ${ }^{20}$. No obstante, al iniciar la terapia, en más de la mitad de los pacientes se puede observar un flare inicial en el cintigrama óseo, cuya interpretación debe ser cautelosa pues no necesariamente traduce progresión de la enfermedad ${ }^{21}$.

En el estudio complementario (COU-AA-302) se evaluó el uso de AA/prednisona en pacientes con CPmRC previo a terapias citotóxicas (docetaxel); 1.088 pacientes asintomáticos o con síntomas mínimos fueron aleatoriamente asignados (en razón 1:1) a AA/prednisona versus placebo/ prednisona con las mismas dosis que en el estu- dio post docetaxel. Luego del segundo análisis interino ocurridos $43 \%$ de los eventos esperados con una mediana de seguimiento de 22,2 meses, el estudio fue abierto al constatarse beneficios significativos de AA/prednisona en sobrevida global (mediana no alcanzada vs 27,2 meses; HR 0,75: IC 95\% 0,61-0,93; $p=0,01$ ) y sobrevida libre de progresión radiológica ( 16,5 vs 8,3 meses; HR 0,53: IC 95\% 0,45-0,62; $\mathrm{p}<0,001)^{22}$. En un análisis reciente del estudio con seguimiento medio de 27,1 meses, los beneficios en sobrevida libre de progresión y global se mantuvieron (Tabla 1), sin embargo, esta última no cruzó la barrera de significancia preestablecida en el diseño del estudio ( $\mathrm{p}<0,0035)$. Consistentemente, todas 


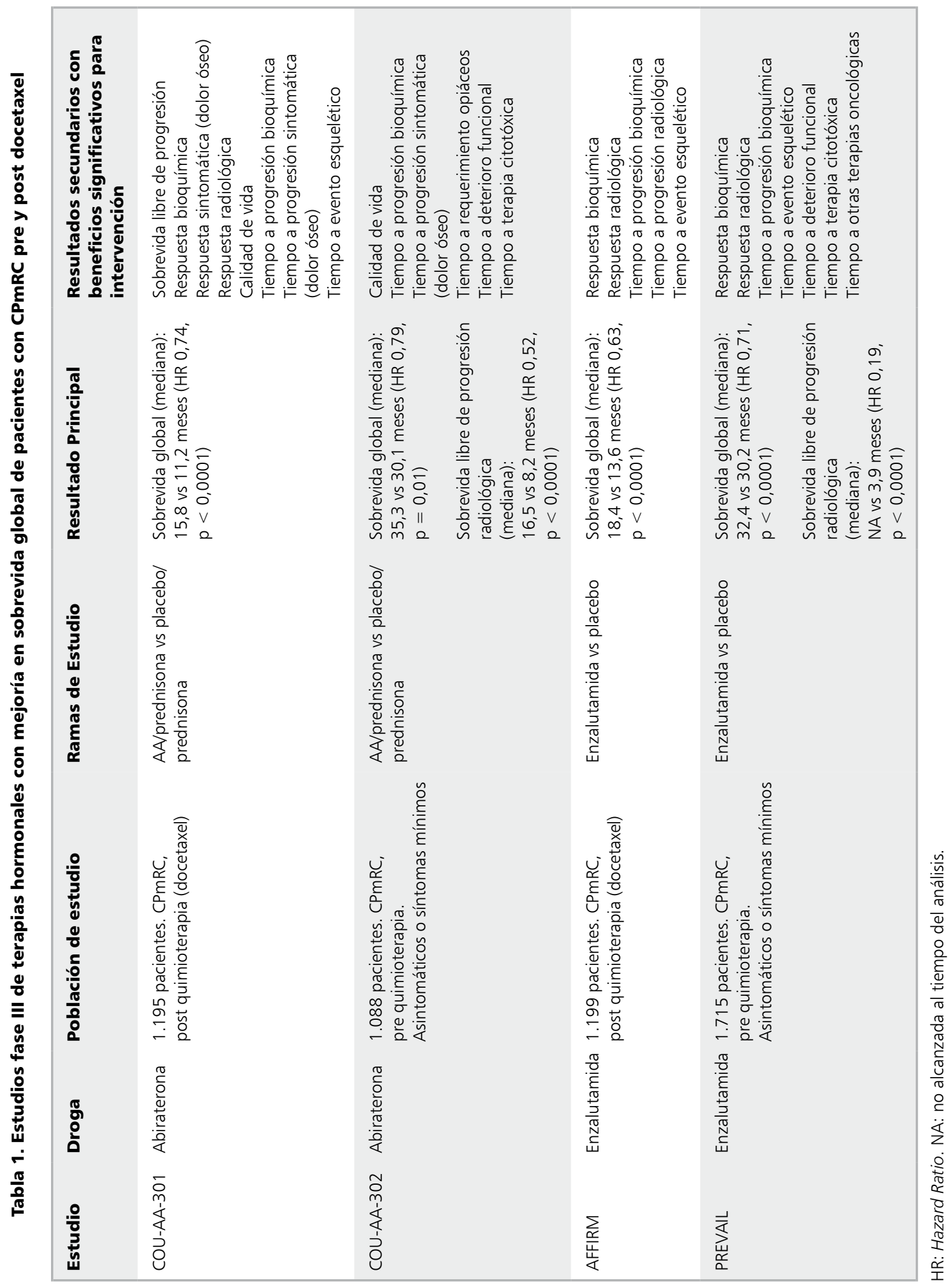


las variables secundarias favorecieron el grupo de intervención AA/prednisona ${ }^{23,24}$.

Los principales efectos secundarios de la terapia con $\mathrm{AA} /$ prednisona observados en estos estudios fase III se resumen en la Tabla 2. Es importante destacar que estos efectos secundarios llevaron a interrumpir la terapia en sólo $10 \%$ de los casos (comparado con $9 \%$ en grupo placebo). Más aún, los perfiles e incidencias de efectos secundarios son similares en pacientes jóvenes y mayores ( $>75$ años) ${ }^{25}$.

De alta relevancia para países con escasos recursos o planes de cobertura limitada para nuevas terapias son los estudios evaluando diferentes dosificaciones de AA. La dosis recomendada y aprobada actualmente es $1.000 \mathrm{mg}$ al día tomada con estómago vacío. Debido a su liposolubilidad, la coadministración con alimentos aumenta la absorción de la droga ${ }^{26,27}$. En experiencia preliminar del Princess Margaret Cancer Centre, AA 250-500 mg al día ingeridos junto a comidas, tendría resultados clínicos equivalentes al grupo de pacientes con la prescripción estándar ${ }^{28}$. Un estudio prospectivo fase II con caracterización farmacodinámica y potencial impacto clínico de dosis reducida está actualmente en curso ${ }^{29}$.

\section{Enzalutamida: receptor androgénico como blanco terapéutico}

Enzalutamida es un antagonista selectivo de alta afinidad del RA. Sus mecanismos de acción inhibitorios son múltiples y a varios niveles en la transducción de señal androgénica: unión competitiva al RA, reducción de la translocación nuclear del RA, interferencia en la unión a factores de transcripción y/o reclutamiento de coactivadores del RA, supresión de la proliferación celular e inducción de apoptosis ${ }^{30}$. A diferencia de otros antiandrogénicos (bicalutamida) carece de actividad agonista residual sobre el RA. Enzalutamida no requiere coadministración de corticoesteroides pues no afecta el eje hipotalámico-hipofisario-adrenal. En el estudio fase I/II se observaron auspiciosas respuestas bioquímicas tanto en el escenario pre como post docetaxel, fijando la dosis terapéutica en $160 \mathrm{mg}$ al día, luego de observar incidencia de convulsiones con dosis mayores ${ }^{31}$.

El estudio fase III AFFIRM, evaluó el beneficio de enzalutamida en pacientes con CPmRC previamente tratados con docetaxel (Tabla 1). En forma controlada, aleatoria y doble-ciego,

Tabla 2. Dosificación y efectos secundarios de la terapia con abiraterona y enzalutamida

\begin{tabular}{|c|c|c|c|}
\hline Droga & Dosificación & $\begin{array}{l}\text { Principales efectos secundarios } \\
(\text { G1-G4)* }\end{array}$ & $\begin{array}{l}\text { Principales efectos secundarios } \\
(\text { G3/G4)\# }\end{array}$ \\
\hline $\begin{array}{l}\text { Abiraterona } \\
\left(\text { Zytiga }{ }^{\circledR}\right)^{\wedge}\end{array}$ & $\begin{array}{l}1.000 \text { mg una } \\
\text { vez al día } \\
\text { Vía oral con } \\
\text { estómago vacío } \\
\text { Se co-administra } \\
\text { con Prednisona } \\
\text { ( } 5 \text { mg dos veces } \\
\text { al día) }\end{array}$ & $\begin{array}{l}\text { Fatiga } 39-47 \%(34-44) \\
\text { Edema } 28-33 \%(22-24) \\
\text { Náusea } 22-33 \%(22-33) \\
\text { Dolor músculo-esquelético 20-33\% (19-36) } \\
\text { Constipación 23-28\% (19-32) } \\
\text { Diarrea 18-22\% (14-18) } \\
\text { Anemia 23-25\% (26-28) } \\
\text { Hipokalemia 17-18\% (9-13) } \\
\text { Alteración pruebas hepáticas 10-12\% (5-9) } \\
\text { Hipertensión 10-22\% (8-13) }\end{array}$ & $\begin{array}{l}\text { Fatiga 8-9\% (10) } \\
\text { Anemia 7-8\% (8-9) } \\
\text { Dolor músculo-esquelético 4-7\% (4-11) } \\
\text { Alteración pruebas hepáticas 3-5\% (3) }\end{array}$ \\
\hline $\begin{array}{l}\text { Enzalutamida } \\
(\text { Xtandi } \circledast)^{*}\end{array}$ & $\begin{array}{l}160 \text { mg una vez } \\
\text { al día } \\
\text { Vía oral }\end{array}$ & $\begin{array}{l}\text { Fatiga } 34 \%(29) \\
\text { Diarrea } 21 \%(18) \\
\text { Dolor músculo-esquelético 15-26\% (11-24) } \\
\text { Bochornos } 20 \%(10) \\
\text { Edema } 15 \%(13) \\
\text { Cefalea } 12 \%(6)\end{array}$ & $\begin{array}{l}\text { Fatiga } 6 \%(7) \\
\text { Convulsiones secundarias < 1\%(0) }\end{array}$ \\
\hline
\end{tabular}

*Incidencia de efectos secundarios basado sólo en un estudio (PREVAIL), que reportó toxicidades significativas respecto a grupo placebo. ^ Rango de efectos secundarios combinado de estudios COU-AA-301 y 302. "Se muestran sólo efectos secundarios significativos o incidencia $>5 \%$. Paréntesis muestra \% toxicidad en grupo placebo respectivo. 
1.199 hombres fueron asignados (en razón 2:1) a enzalutamida versus placebo. Se incluyeron pacientes con confirmación histológica de cáncer de próstata, en niveles hormonales de castración (testosterona $<50 \mathrm{ng} / \mathrm{dL}$ ) y buen estado funcional (ECOG PS 0-2). La variable principal del estudio fue sobrevida global, con variables secundarias de progresión, respuestas y calidad de vida. El tratamiento se mantuvo hasta la progresión (clínica o radiológica) y/o tres incrementos consecutivos del APE. Luego del análisis interino, la rama activa demostró un incremento medio en sobrevida global de 4,8 meses $(p<0,0001)^{32}$. De forma similar a lo ocurrido con AA, el Comité de Monitorización determinó la apertura del estudio para permitir el cruce del grupo placebo a tratamiento activo. Un análisis reciente, confirmó equivalencia en los beneficios en el subgrupo de pacientes mayores (> 75 años) comparados con pacientes jóvenes, con sólo incrementos mínimos de fatiga y efectos secundarios de bajo grado ${ }^{33}$.

Evaluar el uso de enzalutamida en estadios más precoces (CPmRC pre docetaxel) es el objetivo del estudio randomizado fase III PREVAIL (Tabla 1). Los resultados iniciales fueron presentados recientemente ${ }^{34,35}$; estando pendientes los definitivos. Pacientes asintomáticos o con síntomas mínimos fueron randomizados (en razón 1:1) a enzalutamida versus placebo. La variable primaria fue la combinación de sobrevida global y libre de progresión radiológica. Nuevamente, en el análisis interino los beneficios fueron evidentes en la variables principales (reducción riesgo de muerte en 30\%; p $<0,0001)$ y en todas las variables secundarias. Similar a lo ocurrido en los estudios previamente mencionados, el Comité de Monitorización recomendó la apertura del ciego del estudio considerando la eficacia y beneficios evidentes de enzalutamida en este escenario clínico. Destacable fue la tasa de respuesta bioquímica (reducción APE > 50\%) en aproximadamente $80 \%$ de los pacientes, siendo ésta significativa (reducción $>90 \%$ ) en más de la mitad de los casos. Enzalutamida incrementó asimismo el tiempo medio en requerir terapia citotóxica de 10,8 a 28 meses $(\mathrm{p}<0,0001)$.

Los principales efectos secundarios de la terapia con enzalutamida se resumen en la Tabla 2. La interrupción de la terapia por toxicidad fue un hecho infrecuente ( $8 \%$ de los casos, comparado con $10 \%$ en grupo placebo).

\section{Otras terapias hormonales}

Orteronel (TAK-700), similar a AA, se diferencia por una menor inhibición de 17a-hidroxilasa, por lo tanto, teóricamente no requeriría coadministración de prednisona ${ }^{36}$. Sin embargo, dos estudios randomizados ${ }^{37,38}$ evalúan su uso en pacientes con CPmRC pre y post docetaxel combinando orteronel/prednisona obviando la ventaja teórica por sobre AA. En una comunicación reciente, el uso de orteronel/prednisona demostró beneficio sólo en sobrevida libre de progresión, sin impacto en sobrevida global ${ }^{39}$. El acceso a AA y enzalutamida con impacto demostrado en sobrevida, puede ser un factor confundente en estos resultados, lo cual deberá corroborarse a futuro ${ }^{40}$.

Basado en el mecanismo y éxito de enzalutamida, ARN-509 es otro nuevo antagonista del RA. Estudios preclínicos y clínicos iniciales sugieren mayor actividad e índice terapéutico, buen perfil de seguridad y tasas/duración de respuestas promisorias ${ }^{41,42}$. ARN-509 versus placebo en CPRC no metastásico $(S P A R T A N)^{43}$ y el uso conjunto con $\mathrm{AA}$ en $\mathrm{CPmRC}^{44}$ son estudios actualmente en curso. Otro antiandrogénico de última generación, ODM-201, tendría la ventaja de no penetrar la barrera hematoencefálica ${ }^{45}$, una de las principales aprehensiones con enzalutamida, a pesar de la observación infrecuente de convulsiones secundarias $(<1 \%)$.

Finalmente, la maximización de la terapia con un fármaco que combine la inhibición enzimática CYP-17 y del RA es la lógica detrás del desarrollo de TOK- $001^{46}$, actualmente en fases iniciales de evaluación ${ }^{47}$.

\section{Otras terapias con impacto en sobrevida global}

Existen otros fármacos evaluados en estudios fase III que han demostrado beneficios significativos en el manejo del CPmRC (Tabla 3). En pacientes con progresión post docetaxel, el uso de cabazitaxel (Taxano de segunda generación) ${ }^{48}$ ha demostrado beneficios en sobrevida global y 


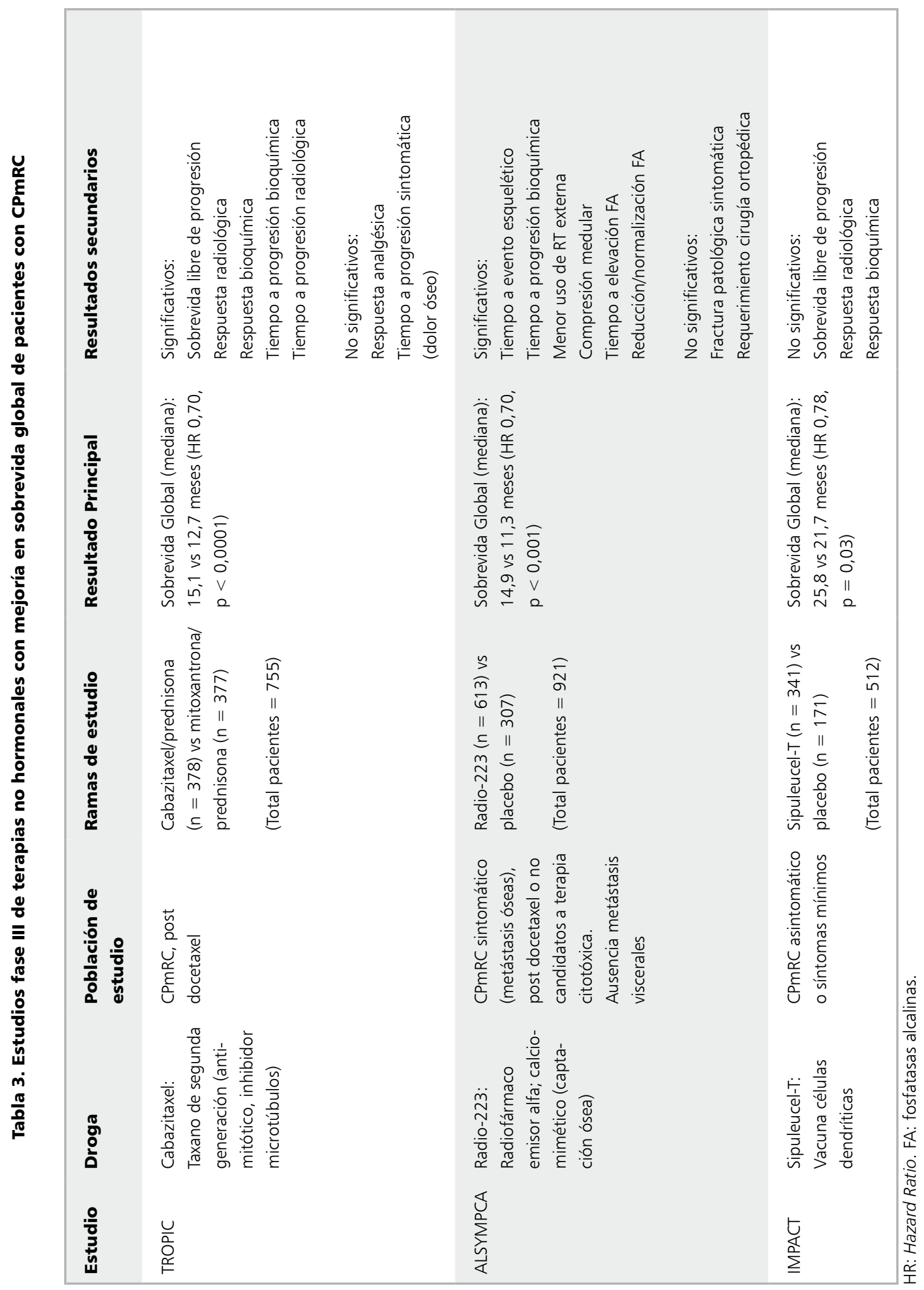


respuestas de la enfermedad (radiológicas y bioquímicas). Sin embargo, no existirían mejorías significativas en términos de respuesta analgésica o en el tiempo de progresión sintomática relacionada a metástasis óseas ${ }^{49}$. Una de las consideraciones importantes es la mortalidad atribuible al tratamiento (5\% versus $1,9 \%$ rama control mitoxantrona/prednisona), principalmente asociada a la alta incidencia de neutropenia. En un análisis posterior, el uso profiláctico adecuado y oportuno de factores estimulantes (GCSF) demostró beneficio preventivo significativo en estos pacientes ${ }^{50}$. Estudios actualmente en curso comparan docetaxel versus cabazitaxel como tratamiento citotóxicos de primera línea ${ }^{51}$, además de evaluar eficacia y toxicidad al reducir dosis de cabazitaxel $\left(20 \mathrm{mg} / \mathrm{m}^{2}\right.$ versus $25 \mathrm{mg} / \mathrm{m}^{2}$ cada 3 semanas) ${ }^{52}$.

Radio-223 es un radiofármaco emisor alfa y calcio mimético que se incorpora en metástasis óseas, particularmente osteoblásticas. A diferencia de samario-153 y estroncio-89 (emisores beta), la emisión alfa deposita mayor energía con menor alcance, limitando así la dosis a tejidos sanos circundantes ${ }^{53}$. En pacientes con enfermedad metastásica ósea sintomática, previamente tratados con docetaxel o no candidatos para terapia citotóxica, el uso de radio-223 demostró mejorías en sobrevida global y algunas de las variables secundarias relacionadas a las lesiones óseas. Sin embargo, la incidencia de fractura patológica o necesidad de cirugía ortopédica no fueron reducidas en forma significativa. El perfil de toxicidad fue favorable, observándose mayor incidencia de diarrea ( $25 \%$ vs $15 \%$ en grupo placebo) y baja toxicidad hematológica $(<3 \%$ G3G4 $)^{54}$. Para pacientes con características similares a aquellos incluidos en el estudio ALSYMPCA, radio-223 constituye una alternativa terapéutica adicional al manejo estándar que incluyó terapia hormonal, analgesia, bisfosfonatos y radioterapia externa de acuerdo a las indicaciones y beneficios demostrados ${ }^{55}$.

Sipuleucel-T es una vacuna basada en células dendríticas, constituyendo el primer tratamiento inmunológico en ser aprobado para el CPmRC. Un análisis combinado de dos estudios fase III pequeños (D9901 y D9902A) negativos para la variable principal (sobrevida libre de progresión) sugirió un beneficio potencial en sobrevida global ${ }^{56}$. Este beneficio se confirmó en el estudio
IMPACT en pacientes con CPmRC asintomático o con síntomas mínimos, hecho que fue fundamental para lograr la aprobación de este tipo de terapia. Sin embargo, ninguna de las variables secundarias de respuesta clínica demostró mejoras significativas. Asimismo, el mecanismo tras el beneficio no es claro; si bien la vacuna logró aumentar los títulos de anticuerpos contra el inmunógeno recombinante (PA2024), no hubo correlación entre los anticuerpos contra la fosfatasa ácida prostática y los beneficios observados ${ }^{57}$. Los costos de la terapia, la complejidad y los tiempos de aféresis y preparación de la vacuna, la falta de beneficio en las variables tradicionales (sobrevida libre de progresión y respuestas bioquímicas o radiológicas) son algunas de las razones para la lenta adopción de esta terapia ${ }^{58}$. Mejor entendimiento del mecanismo de acción (y variables adecuadas para objetivar los resultados) de este tipo de terapias, sumado a biomarcadores que identifiquen pacientes con mayor probabilidad de beneficiarse, serán fundamentales para incrementar el uso de inmunoterapia en el arsenal terapéutico actual. Si bien, los recientes resultados de ipilimumab (anti CTLA-4) en CPmRC postdocetaxel fueron decepcionantes al no mostrar beneficio en sobrevida global ${ }^{59}$, es esperable que la inmunoterapia en CP continúe siendo un área de activo desarrollo.

\section{Futuro: secuenciación y/o sinergia}

Paradójicamente, los rápidos avances terapéuticos han enfrentado a la comunidad médica con la dificultad de definir la secuenciación óptima de estas terapias. Reportes iniciales parecen coincidir que las respuestas a AA post enzalutamida (o viceversa) son menores que al tratamiento primario ${ }^{60-63}$. Agregando mayor complejidad a la elección de tratamiento inicial, estudios preclínicos sugieren cierta superposición en los mecanismos de acción y por lo tanto, resistencia cruzada parcial entre taxanos y hormonoterapia $^{64-65}$, fenómeno que sería menor en el caso de cabazitaxel ${ }^{66}$. Concordante con esta evidencia, series clínicas han demostrado menores tasas de respuesta a docetaxel en pacientes previamente tratados con hormonoterapia de segunda generación ${ }^{65,67}$. Sin embargo, este fenómeno no ha sido observado aún con cabazitaxel ${ }^{68-70}$. El 
corolario de esta evidencia se resume en que la terapia inicial del CPmRC podría comprometer las tasas de respuesta a los tratamientos de segunda y tercera línea. Desafortunadamente, en la actualidad no existen estudios prospectivos evaluando la secuencia óptima de tratamientos y su realización se vislumbra como poco probable. En forma consistente, las recomendaciones y guías clínicas recientes no han consensuado respecto a la terapia inicial de elección para el CPmRC $\mathrm{C}^{71-73}$. Considerando la heterogeneidad inherente de la enfermedad y en las respuestas a los distintos tratamientos, la identificación y validación de biomarcadores será fundamental. Biomarcadores predictivos de respuesta a tratamientos o para medición precoz de eficacia, permitirán individualizar, guiar y objetivar las elecciones de terapias, optimizando así los resultados globales del tratamiento ${ }^{74}$. En contraste con la terapia secuencial, los diferentes mecanismos de acción de AA y enzalutamida sustentan la hipótesis de sinergismo entre estas terapias. Series pequeñas recientes han confirmado resultados promisorios $\mathrm{y}$ ausencia de toxicidades inesperadas con el uso combinado ${ }^{75}$. De hecho, estudios fase III de esta combinación están en curso ${ }^{76}$.

En la actualidad, la elección para cada caso particular debe basarse principalmente en la accesibilidad a los medicamentos, costos, evaluación de la evidencia actual, criterios de inclusión de los estudios y los perfiles de toxicidad específicos de cada droga ${ }^{77}$. Algunos elementos adicionales que podrían ser utilizados para favorecer el uso inicial de hormonoterapia son la respuesta y duración al tratamiento inicial de castración ${ }^{78}$, tumores de grado histológico (Gleason) bajo o intermedio ${ }^{79}$, síntomas leves-moderados, ausencia o escasa enfermedad visceral, riesgo elevado de complicaciones con terapia citotóxica, entre otros.

Un área de activa investigación es el surgimiento de resistencia a las subsecuentes líneas de terapia. Diferenciar posibles procesos de adaptación adquiridos durante la terapia versus selección y emergencia de clones resistentes permitirá definir mejor los esquemas terapéuticos para sobrellevar dichos mecanismos y mejorar los resultados. En este escenario, cobra relevancia la caracterización genómica del CP. Estudios referentes en $\mathrm{CPmRC}$ han mostrado alteraciones recurrentes en ciertos genes pertenecientes a vías de señalización celular complementarias (ej.
AR, MYC, PTEN, SPOP, MLL2) ${ }^{80}$. Asimismo, se han detectado otras alteraciones de menor frecuencia para las cuales hay medicamentos disponibles (ej. vía de señalización PI3K/AKT/ mTOR, vía de señalización RAS/RAF, DNAPK, PARP, AURKA) $)^{81}$. Mejor entendimiento de los mecanismos de adquisición y evolución de las alteraciones genómicas del $\mathrm{CP}^{82}$ junto a mayor caracterización molecular del CPmRC sentarán sin duda nuevas bases para entender los mecanismos de resistencia a las nuevas terapias, el descubrimiento de nuevos blancos terapéuticos y finalmente lograr la selección óptima de tratamiento para cada caso particular, en la era de la medicina personalizada ${ }^{83}$.

\section{Conclusiones}

Las comodidades de administración y el perfil de seguridad hacen de abiraterona y enzalutamida fármacos extremadamente atractivos en una enfermedad que se había caracterizado por más de seis décadas de avances terapéuticos marginales. La incorporación de ellas al arsenal terapéutico y su uso por una amplia gama de especialistas relacionados al diagnóstico y manejo del CP (urólogos, oncólogos radioterapeutas y oncólogos médicos) puede traducir los excelentes resultados de estudios clínicos en una realidad para los pacientes que enfrentan esta enfermedad y que luego del fracaso de terapias con intención curativa (radioterapia y/o cirugía) son habitualmente catalogados como incurables. En los próximos años, se espera además un mayor entendimiento respecto al rol de estos agentes en estadios más tempranos (curables) de la enfermedad. Por otro lado, un mayor conocimiento y comprensión del genoma del CPmRC y la evolución de éste durante las terapias, permitirán la selección personalizada de esquemas terapéuticos óptimos para las distintas fases de la enfermedad.

Se anticipa la necesidad de análisis y estudios de aplicabilidad, accesibilidad y costos de estas nuevas terapias en nuestro país y el resto de Latinoamérica para lograr su incorporación al arsenal terapéutico actual. No obstante, la familiarización de la comunidad médica nacional con estos nuevos agentes es imperativa para traducir los beneficios demostrados en un impacto positivo para los pacientes con $\mathrm{CP}$. 


\section{Referencias}

1. Ferlay J, Shin HR, Bray F, Forman D, Mathers C, Parkin DM. GLOBOCAN 2008 v2.0, Cancer Incidence and Mortality Worldwide: IARC CancerBase No. 10. Lyon, France: International Agency for Research on Cancer. 2010. Disponible en http://globocan.iarc.fr. [Consultado el 07 de mayo de 2014].

2. Departamento de Estadística e Información en Salud 2011. Ministerio de Salud. Gobierno de Chile. Disponible en: www.deis.cl. [Consultado el 07 de mayo de 2014].

3. Huggins C, Hodges CV. Studies on prostatic cancer: I. The effect of castration, of estrogen and of androgen injection on serum phosphatases in metastatic carcinoma of the prostate. Cancer Res 1941; 1: 293-7.

4. Eisenberger MA, Blumenstein BA, Crawford ED, Miller G, McLeod DG, Loehrer PJ, et al. Bilateral orchiectomy with or without flutamide for metastatic prostate cancer. N Engl J Med 1998; 339 (15): 1036-42.

5. Crook JM, O'Callaghan CJ, Duncan G, Dearnaley DP, Higano CS, Horwitz EM, et al. Intermittent androgen suppression for rising PSA level after radiotherapy. N Engl J Med 2012; 367 (10): 895-903.

6. Tannock IF, Osoba D, Stockler MR, Ernst DS, Neville AJ, Moore MJ, et al. Chemotherapy with mitoxantrone plus prednisone or prednisone alone for symptomatic hormone-resistant prostate cancer: a Canadian randomized trial with palliative end points. J Clin Oncol 1996; 14 (6): 1756-64.

7. Kantoff PW, Halabi S, Conaway M, Picus J, Kirshner J, Hars V, et al. Hydrocortisone with or without mitoxantrone in men with hormone-refractory prostate cancer: results of the cancer and leukemia group B 9182 study. J Clin Oncol 1999; 17 (8): 2506-6.

8. Petrylak DP, Tangen CM, Hussain MHA, Lara PN, Jones JA, Taplin M-E, et al. Docetaxel and estramustine compared with mitoxantrone and prednisone for advanced refractory prostate cancer. N Engl J Med 2004; 351 (15): 1513-20.

9. Tannock IF, de Wit R, Berry WR, Horti J, Pluzanska A, Chi KN, et al. Docetaxel plus prednisone or mitoxantrone plus prednisone for advanced prostate cancer. $\mathrm{N}$ Engl J Med 2004; 351 (15): 1502-12.

10. Berthold DR, Pond GR, Soban F, de Wit R, Eisenberger $\mathrm{M}$, Tannock IF. Docetaxel plus prednisone or mitoxantrone plus prednisone for advanced prostate cancer: updated survival in the TAX 327 study. J Clin Oncol 2008; 26 (2): 242-5.

11. Horwich A, Hugosson J, de Reijke T, Wiegel T, Fizazi $\mathrm{K}$, Kataja V, et al. Prostate cancer: ESMO Consensus
Conference Guidelines 2012. Ann Oncol 2013; 24 (5): 1141-62.

12. Kavallaris M. Microtubules and resistance to tubulinbinding agents. Nat Rev Cancer 2010; 10 (3): 194-204.

13. O’Neill AJ, Prencipe M, Dowling C, Fan Y, Mulrane $\mathrm{L}$, Gallagher WM, et al. Inactivation of nuclear factor kappaB by soy isoflavone genistein contributes to increased apoptosis induced by chemotherapeutic agents in human cancer cells. Mol Cancer 2011; 10 (15): 6934-42.

14. Chen Y, Clegg NJ, Scher HI. Anti-androgens and androgen-depleting therapies in prostate cancer: new agents for an established target. The Lancet Oncol 2009; 10 (10): 981-91.

15. Azzouni F, Mohler J. Biology of castration-recurrent prostate cancer. Urol Clin North Am 2012; 39 (4): 43552.

16. Leibowitz-Amit R, Joshua AM. Targeting the androgen receptor in the management of castration-resistant prostate cancer: rationale, progress, and future directions. Curr Oncol 2012; 19 (Suppl 3): S22-31.

17. Bryce A, Ryan CJ. Development and clinical utility of abiraterone acetate as an androgen synthesis inhibitor. Clin Pharmacol Ther 2012; 91 (1): 101-8.

18. de Bono JS, Logothetis CJ, Molina A, Fizazi K, North $\mathrm{S}$, Chu L, et al. Abiraterone and increased survival in metastatic prostate cancer. N Engl J Med 2011; 364 (21): 1995-2005.

19. Fizazi K, Scher HI, Molina A, Logothetis CJ, Chi KN, Jones RJ, et al. Abiraterone acetate for treatment of metastatic castration-resistant prostate cancer: final overall survival analysis of the COU-AA-301 randomised, double-blind, placebo-controlled phase 3 study. Lancet Oncol 2012; 13 (10): 983-92.

20. Logothetis CJ, Basch E, Molina A, Fizazi K, North SA, Chi KN, et al. Effect of abiraterone acetate and prednisone compared with placebo and prednisone on pain control and skeletal-related events in patients with metastatic castration-resistant prostate cancer: exploratory analysis of data from the COU-AA-301 randomised trial. Lancet Oncol 2012; 13 (12): 1210-7.

21. Ryan CJ, Shah S, Efstathiou E, Smith MR, Taplin M-E, Bubley GJ, et al. Phase II study of abiraterone acetate in chemotherapy-naive metastatic castration-resistant prostate cancer displaying bone flare discordant with serologic response. Clin Cancer Res 2011; 17 (14): 485461.

22. Ryan CJ, Smith MR, de Bono JS, Molina A, Logothetis CJ, de Souza P, et al. Abiraterone in metastatic prostate cancer without previous chemotherapy. N Engl J Med 2013; 368 (2): 138-48.

23. Basch E, Autio K, Ryan CJ, Mulders P, Shore N, Kheoh T, 
et al. Abiraterone acetate plus prednisone versus prednisone alone in chemotherapy-naive men with metastatic castration-resistant prostate cancer: patient-reported outcome results of a randomised phase 3 trial. Lancet Oncol 2013; 14 (12): 1193-9.

24. Rathkopf DE, Smith MR, de Bono JS, Logothetis CJ, Shore ND, de Souza P, et al. Updated Interim Efficacy Analysis and Long-term Safety of Abiraterone Acetate in Metastatic Castration-resistant Prostate Cancer Patients Without Prior Chemotherapy (COU-AA-302). Eur Urol 2014; Mar 6: Epub ahead of print. [Consultado el 06 de junio de 2014].

25. Mulders PF, Molina A, Marberger M, Saad F, Higano CS, Chi KN, et al. Efficacy and safety of abiraterone acetate in an elderly patient subgroup (aged 75 and older) with metastatic castration-resistant prostate cancer after docetaxel-based chemotherapy. Eur Urol 2014; 65 (5): 875-83.

26. Ryan CJ, Smith MR, Fong L, Rosenberg JE, Kantoff P, Raynaud F, et al. Phase I clinical trial of the CYP17 inhibitor abiraterone acetate demonstrating clinical activity in patients with castration-resistant prostate cancer who received prior ketoconazole therapy. J Clin Oncol 2010; 28 (9): 1481-8.

27. Acharya M, Bernard A, González M, Jiao J, De Vries R, Tran N. Open-label, phase I, pharmacokinetic studies of abiraterone acetate in healthy men. Cancer Chemother Pharmacol 2012; 69 (6): 1583-90.

28. Leibowitz-Amit R, Seah JA, Atenafu EG, Templeton AJ, Vera-Badillo FE, Alimohamed N, et al. Abiraterone acetate in metastatic castration-resistant prostate cancer: A retrospective review of the Princess Margaret experience of (I) low dose abiraterone and (II) prior ketoconazole. Eur J Cancer 2014; 50 (14): 2399-407.

29. National Cancer Institute (NCI); University of Chicago. Food Effect Study of Abiraterone Acetate for Treatment of Patients With Castration-Resistant Prostate Cancer. En: ClinicalTrials.gov [Internet]. Disponible en: http:// clinicaltrials.gov/show/NCT01543776. Identificador NLM: NCT01543776. (Consultado el 06 de junio de 2014).

30. Tran C, Ouk S, Clegg NJ, Chen Y, Watson PA, Arora V, et al. Development of a second-generation antiandrogen for treatment of advanced prostate cancer. Science 2009; 324 (5928): 787-90.

31. Scher HI, Beer TM, Higano CS, Anand A, Taplin M-E, Efstathiou E, et al. Antitumour activity of MDV3100 in castration-resistant prostate cancer: a phase 1-2 study. Lancet 2010; 375 (9724): 1437-46.

32. Scher HI, Fizazi K, Saad F, Taplin M-E, Sternberg CN, Miller $\mathrm{K}$, et al. Increased survival with enzalutamide in prostate cancer after chemotherapy. N Engl J Med 2012; 367 (13): 1187-97.

33. Sternberg CN, de Bono JS, Chi KN, Fizazi K, Mulders P, Cerbone $\mathrm{L}$, et al. Improved outcomes in elderly patients with metastatic castration-resistant prostate cancer treated with the androgen receptor inhibitor enzalutamide: results from the phase III AFFIRM trial. Ann Oncol 2014; 25 (2): 429-34.

34. Beer TM, Armstrong AJ, Sternberg CN, Higano CS, Iversen $\mathrm{P}$, Loriot $\mathrm{Y}$, et al. Enzalutamide in men with chemotherapy-naive metastatic prostate cancer (mCRPC): Results of phase III PREVAIL study. J Clin Oncol 2014; 32 (4s): abstr LBA1.

35. Armstrong AJ, Tombal B, Sternberg CN, Higano CS, Rathkopf DE, Loriot Y, et al. Primary, secondary, and quality-of-life endpoint results from PREVAIL, a phase 3 study of enzalutamide in men with metastatic castration resistant prostate cancer (mCRPC). J Clin Oncol 2014; 32 (5s): abstr 5007.

36. Kaku T, Hitaka T, Ojida A, Matsunaga N, Adachi M, Tanaka T, et al. Discovery of orteronel (TAK-700), a naphthylmethylimidazole derivative, as a highly selective 17,20-lyase inhibitor with potential utility in the treatment of prostate cancer. Bioorg Med Chem 2011; 19 (21): 6383-99.

37. Millennium Pharmaceuticals. Study of TAK-700 in Combination With Docetaxel and Prednisone in Men With Metastatic Castration-Resistant Prostate Cancer. En: ClinicalTrials.gov [Internet]. Disponible en: http:// clinicaltrials.gov/ct2/show/NCT01084655. Identificador NLM: NCT01084655. (Consultado el 06 de junio de 2014).

38. Millennium Pharmaceuticals. Study Comparing Orteronel Plus Prednisone in Patients With Metastatic Castration-Resistant Prostate Cancer. En: ClinicalTrials. gov [Internet]. Disponible en: http://clinicaltrials. gov/ct2/show/NCT01193257. Identificador NLM: NCT01193257. (Consultado el 06 de junio de 2014).

39. de Wit R, Fizazi K, Jinga V, Efstathiou E, Fong PCC, Wirth $\mathrm{M}$, et al. Phase 3, randomized, placebo-controlled trial of orteronel (TAK-700) plus prednisone in patients (pts) with chemotherapy-naïve metastatic castrationresistant prostate cancer (mCRPC) (ELM-PC 4 trial). J Clin Oncol 2014; 32 (5s): abstr 5008.

40. Fizazi K, Jones R, Oudard S, Efstathiou E, Saad F, de Wit $\mathrm{R}$, et al. Regional differences observed in the phase 3 trial (ELM-PC 5) with orteronel (TAK-700) plus prednisone in patients with metastatic castration-resistant prostate cancer (mCRPC) that has progressed during or following docetaxel. J Clin Oncol 2014; 32 (5s): abstr 5042 . 
41. Clegg NJ, Wongvipat J, Joseph JD, Tran C, Ouk S, Dilhas A, et al. ARN-509: a novel antiandrogen for prostate cancer treatment. Cancer Res 2012; 72 (6): 1494-503.

42. Rathkopf DE, Morris MJ, Fox JJ, Danila DC, Slovin SF, Hager JH, et al. Phase I study of ARN-509, a novel antiandrogen, in the treatment of castration-resistant prostate cancer. J Clin Oncol 2013; 31 (28): 3525-30.

43. Aragon Pharmaceuticals. A Study of ARN-509 in Men With Non-Metastatic Castration-Resistant Prostate Cancer (SPARTAN). En: ClinicalTrials.gov [Internet]. Disponible en: http://clinicaltrials.gov/ct2/show/ NCT01946204. Identificador NLM: NCT01946204. (Consultado el 06 de junio de 2014).

44. Aragon Pharmaceuticals. A Study of JNJ-56021927 (ARN-509) and Abiraterone Acetate in Participants With Metastatic Castration-Resistant Prostate Cancer. En: ClinicalTrials.gov [Internet]. Disponible en: http://clinicaltrials.gov/ct2/show/NCT02123758. Identificador NLM: NCT02123758 (Consultado el 06 de junio de 2014).

45. Massard C, James N, Culine S, Jones R, Vuorela A, Mustonen M. An open-label, phase I/II safety, pharmacokinetic, and proof-of concept study of ODM-201 in patients with progressive metastatic castration-resistant prostate cancer (CRPC). Presentado en European Cancer Congress 2013. Abstract 2853. Disponible en: http:// oncologypro.esmo.org/.

46. Bruno RD, Vasaitis TS, Gediya LK, Purushottamachar P, Godbole AM, Ates-Alagoz Z, et al. Synthesis and biological evaluations of putative metabolically stable analogs of VN/124-1 (TOK-001): head to head antitumor efficacy evaluation of VN/124-1 (TOK-001) and abiraterone in LAPC-4 human prostate cancer xenograft model. Steroids 2011; 76 (12): 1268-79.

47. Montgomery RB, Eisenberger MA, Rettig M, Chu F, Pili $\mathrm{R}$, Stephenson J, et al. Phase I clinical trial of galeterone (TOK-001), a multifunctional antiandrogen and CYP17 inhibitor in castration resistant prostate cancer (CRPC). J Clin Oncol 2012; 30 (suppl): abstr 4665.

48. Mita AC, Denis LJ, Rowinsky EK, Debono JS, Goetz AD, Ochoa L, et al. Phase I and pharmacokinetic study of XRP6258 (RPR 116258A), a novel taxane, administered as a 1-hour infusion every 3 weeks in patients with advanced solid tumors. Clin Cancer Res 2009; 15 (2): 723-30.

49. de Bono JS, Oudard S, Ozguroglu M, Hansen S, Machiels J-P, Kocak I, et al. Prednisone plus cabazitaxel or mitoxantrone for metastatic castration-resistant prostate cancer progressing after docetaxel treatment: a randomised open-label trial. Lancet 2010; 376 (9747): 1147-54.

50. Ozguroglu M, Oudard S, Sartor AO, Hansen S, Machiels $\mathrm{JH}$, Shen L, et al. Effect of G-CSF prophylaxis on the occurrence of neutropenia in men receiving cabazitaxel plus prednisone for the treatment of metastatic castration-resistant prostate cancer (mCRPC) in the TROPIC study. J Clin Oncol 2011: 29 (7s): abstr 144^.

51. Sanofi. Cabazitaxel Versus Docetaxel Both With Prednisone in Patients With Metastatic Castration Resistant Prostate Cancer (FIRSTANA). En: ClinicalTrials.gov [Internet]. Disponible en: http://clinicaltrials.gov/ct2/show/ NCT01308567. Identificador NLM: NCT01308567. (Consultado el 06 de junio de 2014).

52. Sanofi. Cabazitaxel at $20 \mathrm{mg} / \mathrm{m}^{2}$ Compared to $25 \mathrm{mg} /$ $m^{2}$ With Prednisone for the Treatment of Metastatic Castration Resistant Prostate Cancer (PROSELICA). En: ClinicalTrials.gov [Internet]. Disponible en: http://clinicaltrials.gov/show/NCT01308580. Identificador NLM: NCT01308580 (Consultado el 06 de junio de 2014).

53. Liepe K. Alpharadin, a 223Ra-based alpha-particleemitting pharmaceutical for the treatment of bone metastases in patients with cancer. Curr Opin Investig Drugs 2009; 10 (12): 1346-58.

54. Parker C, Nilsson S, Heinrich D, Helle SI, O'Sullivan JM, Fosså SD, et al. Alpha emitter radium-223 and survival in metastatic prostate cancer. N Engl J Med 2013; 369 (3): 213-23.

55. Lutz S, Berk L, Chang E, Chow E, Hahn C, Hoskin P, et al. Palliative radiotherapy for bone metastases: an ASTRO evidence-based guideline. Int J Radiat Oncol Biol Phys 2011; 79 (4): 965-76.

56. Higano CS, Schellhammer PF, Small EJ, Burch PA, Nemunaitis J, Yuh L, et al. Integrated data from 2 randomized, double-blind, placebo-controlled, phase 3 trials of active cellular immunotherapy with sipuleucel- $\mathrm{T}$ in advanced prostate cancer. Cancer 2009; 115 (16): 3670-9.

57. Kantoff PW, Higano CS, Shore ND, Berger ER, Small EJ, Penson DF, et al. Sipuleucel-T immunotherapy for castration-resistant prostate cancer. N Engl J Med 2010; 363 (5): 411-22.

58. Sridhar SS, Freedland SJ, Gleave ME, Higano C, Mulders P, Parker C, et al. Castration-Resistant Prostate Cancer: From New Pathophysiology to New Treatment. Eur Urol 2014; 65 (2): 289-99.

59. Kwon ED, Drake CG, Scher HI, Fizazi K, Bossi A, van den Eertwegh AJM, et al. Ipilimumab versus placebo after radiotherapy in patients with metastatic castrationresistant prostate cancer that had progressed after docetaxel chemotherapy (CA184-043): a multicentre, randomised, double-blind, phase 3 trial. Lancet Oncol 2014; 15 (7): 700-12.

60. Loriot Y, Bianchini D, Ileana E, Sandhu S, Patrikidou A, Pezaro C, et al. Antitumour activity of abiraterone acetate against metastatic castration-resistant prostate 
cancer progressing after docetaxel and enzalutamide (MDV3100). Ann Oncol 2013; 24 (7): 1807-12.

61. Noonan KL, North S, Bitting RL, Armstrong AJ, Ellard SL, Chi KN. Clinical activity of abiraterone acetate in patients with metastatic castration-resistant prostate cancer progressing after enzalutamide. Ann Oncol 2013; 24 (7): 1802-7.

62. Boegemann M, Ohlmann C-H, Schnoeller TJ, Krabbe L-M, Hajili T, Jentzmik F, et al. Enzalutamide in Castration-resistant Prostate Cancer Patients Progressing After Docetaxel and Abiraterone. Eur Urol 2014; 65 (1): 30-6.

63. Bianchini D, Lorente D, Rodríguez-Vida A, Omlin A, Pezaro C, Ferraldeschi R, et al. Antitumour activity of enzalutamide (MDV3100) in patients with metastatic castration-resistant prostate cancer (CRPC) pre-treated with docetaxel and abiraterone. Eur J Cancer 2014; 50 (1): 78-84.

64. van Soest RJ, van Royen ME, de Morrée ES, Moll JM, Teubel W, Wiemer EA, et al. Cross-resistance between taxanes and new hormonal agents abiraterone and enzalutamide may affect drug sequence choices in metastatic castration-resistant prostate cancer. Eur J Cancer 2013; 49 (18): 3821-30.

65. Fitzpatrick JM, de Wit R. Taxane mechanisms of action: potential implications for treatment sequencing in metastatic castration-resistant prostate cancer. Eur Urol 2014; 65 (6): 1198-204.

66. Oudard S, Angelergues A. Prostate cancer: cabazitaxelthe taxane of choice in the new mCRPC landscape? Nat Rev Urol 2014; 11 (7): 370-2.

67. Mezynski J, Pezaro C, Bianchini D, Zivi A, Sandhu S, Thompson E, et al. Antitumour activity of docetaxel following treatment with the CYP17A1 inhibitor abiraterone: clinical evidence for cross-resistance? Ann Oncol 2012; 23 (11): 2943-7.

68. Oudard S, Mercier F, Flechon A, Guillot A, Le Moulec S, Gravis $G$, et al. Efficacy of cabazitaxel and its relationship with predictors of poor response to second hormonal therapies in metastatic castration-resistant prostate cancer (mCRPC). J Clin Oncol 2013; 31 (suppl 6): abstr 137.

69. Pezaro CJ, Omlin AG, Altavilla A, Lorente D, Ferraldeschi R, Bianchini D, et al. Activity of Cabazitaxel in Castration-resistant Prostate Cancer Progressing After Docetaxel and Next-generation Endocrine Agents. Eur Urol 2014; 66 (3): 459-65.

70. Al Nakouzi N, Le Moulec S, Albigès L, Wang C, Beuzeboc P, Gross-Goupil M, et al. Cabazitaxel Remains Active in Patients Progressing After Docetaxel Followed by Novel Androgen Receptor Pathway Targeted Therapies. Eur Urol 2014; May 2: Epub ahead of print. [Consultado el
17 de septiembre de 2014].

71. Heidenreich A, Bastian PJ, Bellmunt J, Bolla M, Joniau $S$, van der Kwast T, et al. EAU guidelines on prostate cancer. Part II: Treatment of advanced, relapsing, and castration-resistant prostate cancer. Eur Urol 2014; 65 (2): 467-79.

72. Fitzpatrick JM, Bellmunt J, Fizazi K, Heidenreich A, Sternberg CN, Tombal B, et al. Optimal management of metastatic castration-resistant prostate cancer: highlights from a European Expert Consensus Panel. Eur J Cancer 2014; 50 (9): 1617-27.

73. Basch E, Loblaw DA, Oliver TK, Carducci M, Chen RC, Frame JN, et al. Systemic Therapy in Men With Metastatic Castration-Resistant Prostate Cancer: American Society of Clinical Oncology and Cancer Care Ontario Clinical Practice Guideline. J Clin Oncol 2014; Sep 8: Epub ahead of print. [Consultado el 17 de septiembre de 2014].

74. Armstrong AJ, Eisenberger MA, Halabi S, Oudard S, Nanus DM, Petrylak DP, et al. Biomarkers in the management and treatment of men with metastatic castration-resistant prostate cancer. Eur Urol 2012; 61 (3): 549-59.

75. Efstathiou E, Titus MA, Wen S, San Miguel A, Hoang A, De Haas- Amatsaleh A, et al. Enzalutamide (ENZA) in combination with abiraterone acetate (AA) in bone metastatic castration resistant prostate cancer (mCRPC). J Clin Oncol 2014; 32 (5s): abstr 5000.

76. Alliance for Clinical Trials in Oncology; National Cancer Institute (NCI). Enzalutamide With or Without Abiraterone Acetate and Prednisone in Treating Patients With Castration-Resistant Metastatic Prostate Cancer. En: ClinicalTrials.gov [Internet]. Disponible en: http://clinicaltrials.gov/show/NCT01949337. Identificador NLM: NCT01949337. (Consultado el 06 de junio de 2014).

77. Bahl A, Masson S, Birtle A, Chowdhury S, de Bono J. Second-line treatment options in metastatic castrationresistant prostate cancer: a comparison of key trials with recently approved agents. Cancer Treat Rev 2014; 40 (1): 170-7.

78. Loriot Y, Massard C, Albiges L, Di Palma M, Blanchard $\mathrm{P}$, Bossi A, et al. Personalizing treatment in patients with castrate-resistant prostate cancer: A study of predictive factors for secondary endocrine therapies activity. J Clin Oncol 2012; 30 (suppl 5): abstr 213.

79. Azria D, Massard C, Tosi D, Houede N, Joly F, Gravis G, et al. An ambispective observational study in the safety and efficacy of abiraterone acetate in the French temporary authorizations for use (ATU): predictive parameters of response. J Clin Oncol 2012; 30 (suppl 5): abstr 149.

80. Grasso CS, Wu Y-M, Robinson DR, Cao X, Dhanase- 
karan SM, Khan AP, et al. The mutational landscape of lethal castration-resistant prostate cancer. Nature 2012; 487 (7406): 239-43.

81. Roychowdhury S, Chinnaiyan AM. Advancing precision medicine for prostate cancer through genomics. J Clin Oncol 2013; 31 (15): 1866-73.

82. Baca SC, Prandi D, Lawrence MS, Mosquera JM, Roma- nel A, Drier Y, et al. Punctuated evolution of prostate cancer genomes. Cell 2013; 153 (3): 666-77.

83. Fraser M, Berlin A, Bristow RG, van der Kwast T. Genomic, pathological, and clinical heterogeneity as drivers of personalized medicine in prostate cancer. Urol Oncol. 2014; Abr 22: Epub ahead of print. [Consultado el 06 de junio de 2014]. 